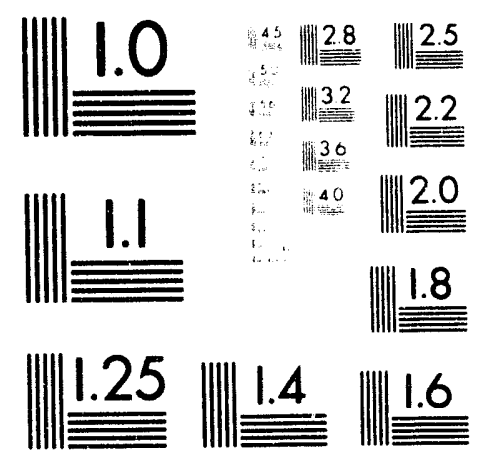



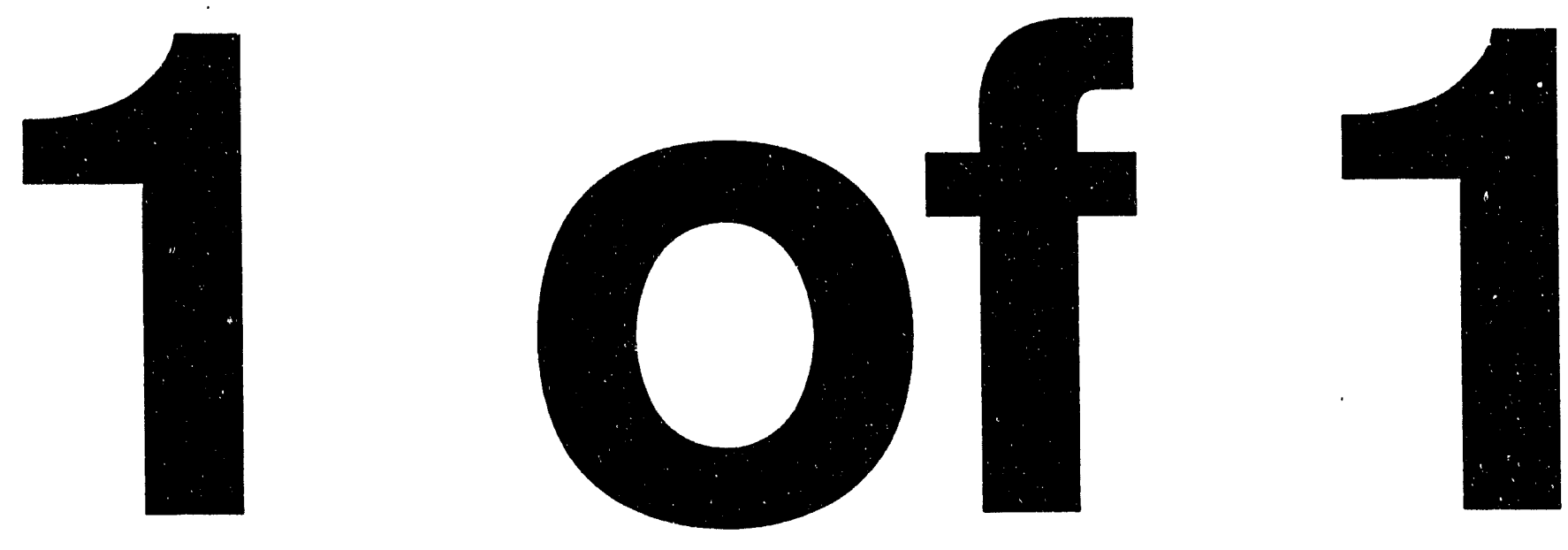


\title{
One- and Multi-Component Theories of Mixtures
}

\author{
Francis H. Ree
}

This paper was prepared for submittal to the Proceedings of the Joint AIRAPT/APS Conference

Colorado Springs, $\mathrm{CO}$

June 28-July 2, 1993

July 1993



\section{DISCLAIMER}

This document was prepared as an account of work sponsored by an agency of the United States Government. Neither the United States Government nor the University of California nor any of their employees, makes any warranty, express or implied, or assumes any legal liability or responsibility for the accuracy, completeness, or usefulness of any information, apparatus, product, or process disclosed, or represents that its use would not infringe privately owned rights. Reference herein to any specific commercial products, process, or service by trade name, trademark, manufacturer, or otherwise, does not necessarily constitute or imply its endorsement, recommendation, or favoring by the United States Government or the University of California. The views and opinions of authors expressed herein do not mecessarily state or reflect those of the United States Government or the University of Califomia, and shall not be used for advertising or product endorsement purposes. 


\title{
ONE- AND MULTI-COMPONENT THEORIES OF MIXTURES
}

\author{
F.ancis H. Ree. \\ Lawrence Livermore National Laboratory \\ University of California \\ P.O. Box 808 \\ Livermore, California 94551, USA
}

We describe one-component statistical mechanical theories and van der Waals's effective one-component mixture model. We then show how to apply them to mixtures (containing $\mathrm{CO}_{2}$ ) to extract reliable unlike-pair potentials involving $\mathrm{CO}_{2}$ molecules as well as their dissociation products. A more fundamental approach will require the development of a perturbation or variational theory of mixtures based on a non-additive hard-sphere mixture reference system. Recent progi esses made in this direction by means of an integral equation and computer simulations is described.

\section{INTRODUCTION}

Main reasons for studying mixtures are: (i) real world is made of mixtures, but at present there is no reliable theory about them; (ii) some physical phenomena, such as fluid-fluid or solid-solid phase separation that can occur only in mixtures need to be understood; (iii) we are interested in finding intermolecular forces among dissimilar molecules.

\section{PURE SYSTEMS}

Understanding the behavior of pure systems is a prerequisite to understanding mixtures. The essential physics behind reliable current models for pure systems owes to van der Waals more than 100 years ago. Van der Waals's idea is that a harsh repulsive portion, rather than the long-range portion, of an intermolecular potential controls thermodynamics and structural properties of condensed phases. Five ingredients are needed to formulate van der Waals's idea into a mathematically rigorous framework:

(a) an optimum way to break an intermolecular potential $V(r)$ into a hard-sphere-like interaction $V_{0}(r)$ and a perturbation term $W(r)$,

$$
V(r)=V_{0}(r)+W(r),
$$

(b) a specific expression for $V_{0}(r)$ and $W(r)$,

(c) an expression for the Helmholtz free energy $A$,

$$
A=A_{0}+A_{1} \text {, }
$$

in terms of the Helmholtz free energy $A_{0}$ for the reference system and that $\left(A_{1}\right)$ for the perturbation potential, (d) an expression for the hard-sphere (HS) diameter to represent the reference potential,

(e) convenient expressions for the HS equation of state (EOS) and radial distribution function $g^{H S}(r)$.

Specific expressions needed for items (a) - (e) are available for classical fluids. ${ }^{1-3}$ One approach is to use our perturbation theory $(\mathrm{PT})^{1}$ which extends earlier theories ${ }^{2}$ to high pressure. The second approach is to employ a variational theory (VAR) developed by Ross. ${ }^{3}$ Both give about equally reliable results and have been successfully extended to describe solidphase thermodynamic properties, 4,5 using analytic expressions 6 for $g^{H S}(r)$. As an example, Table 1 compares $P V / N k T$ ( $P=$ pressure, $V=$ volume, $T=$ temperature; $\rho=N / V)$ data based on the PT and Monte Carlo (MC) simulation data for an exponential$6(\exp -6)$ potential,

$$
V(\dot{r})=\frac{\varepsilon}{(\alpha-6)}\left\{6 \exp \left[\alpha\left(1-r / r^{*}\right)\right]-\alpha\left(r^{*} / r\right)^{6}\right\},
$$

where parameters, $\alpha, \varepsilon$, and $r^{*}$ are specific to each chemical species and can be obtained from experimental data. It is noteworthy that PT is about equally reliable as MC (within MC's statistical errors). Yet, its computation time takes several orders of magnitude less than those required for $\mathrm{MC}$.

Table 1. $P V / N k T$ for an exp-6 potential $(\alpha=13)$.

\begin{tabular}{ccc}
\hline$\rho\left(r^{*}\right)^{3} / \sqrt{2}$ & Monte Carlo & PT \\
\hline 0.9 & 3.27 & 3.27 \\
1.0 & 3.81 & 3.80 \\
1.5 & 8.08 & 8.04 \\
2.05 & 16.58 & 16.65 \\
\hline
\end{tabular}




\section{EFFECTIVE ONE-COMPONENT MIXTURE MODEL}

Compared to like-pair potentials described above, the exp-6 parameters for unlike-pairs are experimentally less well known. But they can be expressed as

$$
\begin{aligned}
& r_{i j}^{\cdot}=k_{i j}\left(\dot{r_{i j}}+r_{i j}^{\cdot}\right) / 2, \\
& \varepsilon_{i j}=l_{i j} \sqrt{\varepsilon_{i i} \varepsilon_{i j}}, \\
& \alpha_{i j}=m_{i j} \sqrt{\alpha_{i j} \alpha_{i j}},
\end{aligned}
$$

in terms of like-pair parameters $\left(r_{i j}^{\cdot}, \varepsilon_{i u}, \alpha_{i j}\right)$ and small multiplicative constants $\left(k_{i}, l_{i j}, m_{i j}\right)$ which can be fixed if appropriate theoretical or experimental data are available. Otherwise, we set $k_{i j}=l_{i j}=m_{i j}=1$ which is commonly referred to as the Lorentz-Berthelot rule.

We assume mixtures of chemical species $i$ and $j$, interacting with exp-6 potentials [Eqs. (3) and (4)] to be a hypothetical one-component system interacting with a composition $\left(x_{i}\right)$-dependen. $2 x p-6$ parameters, ${ }^{7}$

$$
\begin{aligned}
& \left(r^{*}\right)^{3}=\sum_{i \geq j 21}^{n} x_{i} x_{j}\left(r_{i j}^{*}\right)^{3}, \\
& (\varepsilon)^{3}=\sum_{i 2 j 21}^{n} x_{i} x_{j} \varepsilon_{i j}\left(r_{i j}^{*}\right)^{3} /\left(r^{*}\right)^{3}, \\
& (\alpha)^{3}=\sum_{i 2 j 21}^{n} x_{i} x_{j} \varepsilon_{i j} \alpha_{i j}\left(r_{i j}^{*}\right)^{3} / \varepsilon\left(r^{*}\right)^{3} .
\end{aligned}
$$

The fact that one-component calculations are much simpler than mixture calculations provides a reasonable justification for using this so-called improved van der Waals one-fluid model. In fact, comparisons with two-component $\mathrm{MC}$ for $\mathrm{He}-\mathrm{H}_{2}$ mixtures in Table 2 shows that the effective onecomponent model (using VAR) produces excellent results at high pressure and temperature.

Table 2. Pressure (GPa) of an equimular mixture of $\mathrm{H}_{2}$ and $\mathrm{He}$.

\begin{tabular}{rclc}
\hline \hline$T(K)$ & $\begin{array}{c}\mathrm{V} \\
\left(\mathrm{cm}^{3} / \mathrm{mol}\right)\end{array}$ & $\begin{array}{c}\text { 2-component } \\
\text { Monte Carlo }\end{array}$ & $\begin{array}{c}\text { Effective 1- } \\
\text { component } \\
\text { theory }\end{array}$ \\
\hline 50 & 20 & $0.047 \pm 0.01$ & 0.05 \\
100 & 14 & $0.338 \pm 0.008$ & 0.35 \\
300 & 10 & $1.856 \pm 0.02$ & 1.91 \\
4000 & 8 & $12.43 \pm 0.2$ & 12.6 \\
7000 & 4.5 & $54 \pm 1$ & 55.3 \\
\hline \hline
\end{tabular}

\section{APPLICATION TO SHOCK DISSOCIATION OF $\mathrm{CO}_{2}$}

Motivations behind studying unlike-pai: interactions in condensed phases are: (a) these data are unavailable; (b) they can, however, be extracted from strong shock wave data showing shock-induced chemistry; (c) one can study a fluid phase separation at high $P \& T$ as recently done for $\mathrm{N}_{2}+\mathrm{H}_{2} \mathrm{O}$ mixtures. ${ }^{8}$ As an example, we show below how to extract unlike-pair interactions from the recent shock data ${ }^{9}$ of liquid $\mathrm{CO}_{2}$.

A strong shock forres $\mathrm{CO}_{2}$ molecules to partially dissociate into $\mathrm{O}, \mathrm{O}_{2}, \mathrm{CO}$, and carbon residues in the graphitic, diamond, or liquid phase. We assume these dissociation products and $\mathrm{CO}_{2}$ to establish a chemical equilibrium. Their chemical concentrations $\left\{n_{i}\right\}$ will change with $T$ and $P$ and have to be determined by minimizing the Gibbs free energy $G\left(P, T,\left\{n_{i}\right\}\right)$,

$$
G\left(P, T,\left\{n_{i}\right\}\right)=\sum_{i} n_{i} \mu_{i},
$$

with respect to $\left(n_{i}\right)$. The connection between the statistical mechanical theories discussed above (including interaction potentials $V_{i j}(r)$ ) and $G\left(P^{n}, T,\left(n_{i}\right\}\right)$ occurs strictly through the chemical potentials $\mu_{i}$. The minimization is done using the CHEQ code, 10 which evaluates $\mu_{i}$ using the variational theory and the effective one-component mixture model.

For this purpose, we need to know interactions of dissociation products involving single elements $(\mathrm{O}-\mathrm{O}$, $\mathrm{CO}_{2}-\mathrm{O}, \mathrm{O}_{2}-\mathrm{O}_{2}$ ), molecule-molecule interactions ( $\mathrm{CO}$ $\mathrm{CO}, \mathrm{CO}_{2}-\mathrm{CO}_{2}, \mathrm{CO}_{2}-\mathrm{O}_{2}, \mathrm{O}_{2}-\mathrm{CO}$ ), and molecule-atom and atom-atom interactions ( $\mathrm{CO}-\mathrm{O}, \mathrm{CO}_{2}-\mathrm{O}$ ). Among these nine interactions, only three like-pair interactions $\left(\mathrm{O}_{2}-\mathrm{O}_{2}, \mathrm{CO}-\mathrm{CO}, \mathrm{CO}_{2}-\mathrm{CO}_{2}\right)$ are directly determinable from experiment based on onecomponent systems. The rest have to be determined by adjusting $\left(k_{i j}, l_{i j}, m_{i j}\right)$ parameters in Eq. (4) to fit the shock data of liquid $\mathrm{CO}_{2}$. Figure 1 shows the Hugoniot of liquid $\mathrm{CO}_{2}$ obtained by adjusting the interactions parameters described above. They are compared with the experimental data. ${ }^{9}$ As shock pressures increase, $\mathrm{CO}_{2}$ will dissociate into $\mathrm{CO}$ and $\mathrm{O}$ species. At pressures above $40 \mathrm{GPa}$, our calculation predicts that a significant proportion of carbon atoms will condense into the liquid phase,

$$
\mathrm{CO}_{2} \rightarrow \mathrm{CO}+\mathrm{O} \rightarrow \text { liquid } \mathrm{C}+\mathrm{CO}+\mathrm{O} \text {. }
$$

It gives raise to a shoulder-like structure seen in the experimental data near $40 \mathrm{GPa}$. The same set of interaction parameters also gives a satisfactory 


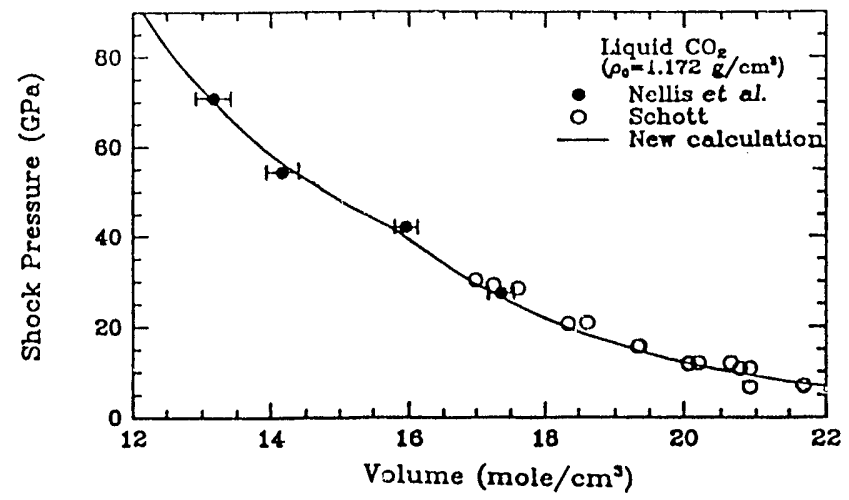

Fig. 1. Theoretical and experimental Hugoniots of liquid $\mathrm{CO}_{2}$

Hugoniot for liquid CO.11 Such an additional test provides a strong self-consistency criterion for unlikepair potentials determined in this manner.

\section{IMPROVED MIXTURE MODEL}

The next generation mixture model requires replacement of the effective one-component approximation, Eq. (5), by a multi-component mixture model. To do this, we need items (a) to (e) (described earlier for pure systems) for mixtures. One major bottleneck in this long-range research project is item (e), i.e., lack of convenient expressions for the HS mixture EOS and the HS mixture radial distribution function $g_{i j}^{H S}(r)$. The former is needed to represent the reference system and the latter for evaluation of the perturbation correction $A_{1}$ and also the HS diameter $d_{i j}$.

There are a large number of independent variables to consider for the HS mixture EOS, i.e., density $\rho$, both like- $(i=j)$, and unlike- $(i \neq j)$ pair HS diameters defined by $\left\{d_{i j}=\left(d_{i j}+d_{i j}\right)\left(1+\alpha_{i j}\right) / 2\right\}$, and species composition $\left\{x_{i}\right\}$. The use of non-zero nonadditivity parameters $\left\{\alpha_{i j}\right\}$ is closer to nature and is needed to describe compound forming alloys or phase separations.

The EOS of pure HS is satisfactorily described by the Carnahan-Starling expression, 12 and that for the additive HS (AHS) mixtures by the MansooriCarnahan-Starling-Leland form. ${ }^{13}$ Our goal is to obtain a similar expression for nonadditive HS (NAHS) mixtures. One cost-effective way to do so is to use the modified Martynov-Sarkisov (MMS) integral equation for $g_{i j}^{H S}(r)$ developed by Ballone $e t$ al. 14 They have shown that MMS agrees closely with
MC data for NAHS. Hence, our approach is to generate EOS data with MMS over a large range of $\left\{\eta_{i j}, \alpha_{i j}, x_{i}\right\}$ and find an expression which fits these data. After this is done, we can try to use the same expression to fit computationally more demanding MC data for NAHS systems at sparsely separated values of $\left\{\eta_{i j}, \alpha_{i j}, x_{i}\right\}$.

Table 3 gives $P V / N k T$ obtained using MMS and our preliminary fit ${ }^{15}$ based on an extension of the expression [Eq. (2.6) in Ref. 14] by Ballone et al. for symmetric HS mixtures $\left(d_{11}=d_{22}, x_{1}=x_{2}\right)$. Both lie within $1 \%$ of each other. Further refinement of this work to nonsymmetric case $x_{1} \neq x_{2}$ using MC data on NAHS is in progress and will be reported in due course. 15

Table 3. PV/NkT for symmetric HS mixtures $d_{11}=d_{22}=d_{,} x_{1}=x_{2}$.

\begin{tabular}{ccccc}
\hline$\alpha$ & $\rho d^{3}$ & Fit $^{\mathbf{a}}$ & MMS $^{\mathbf{b}}$ & \%-error \\
\hline 0.05 & 0.1 & 1.261 & 1.262 & -0.08 \\
0.05 & 0.5 & 3.592 & 3.593 & -0.03 \\
0.02 & 0.1 & 1.248 & 1.249 & -0.08 \\
0.02 & 0.7 & 6.083 & 6.059 & 0.39 \\
-0.2 & 0.2 & 1.384 & 1.384 & 0.00 \\
-0.2 & 1.2 & 8.174 & 8.119 & 0.67 \\
-0.5 & 0.4 & 1.616 & 1.615 & 0.06 \\
-0.5 & 1.6 & 8.045 & 8.060 & -0.19 \\
\hline
\end{tabular}

a Fit = least-squares fit to MMS based on a modification of Eq. (2.6) in Ref. 14.

b MMS = modified Martynov-Sarkisov equation [Ref. 14].

\section{CONCLUDING REMARKS}

We have described the one-component effective mixture model and how one can use it to extract unlike-pair potentials in condensed phases. We plan to remove the effective one-component mixture model by a more basic mixture model. One key area in this regard is to obtain a reliable analytic expression for a reference system, i.e., non-additive hard-sphere mixtures. We have developed such an expression for an equal-diameter symmetric case using the MMS integral equation. Our next plan is to extend it to cases with unequal- diameter nonsymmetric cases and to investigate a possible fluid phase separation, all using MC data for NAHS. After this is done, we will be in a strong position to build a truly basic mixture theory. 


\section{ACKNOWLEDGMENTS}

We are grateful to $M$. van Thiel and J.-K. Jung for information pertinent to the present work. This work was done under the auspices of the U.S. Department of Energy by the Lawrence Livermore National Laboratory under contract 7405-ENG-48.

\section{REFERENCES}

[1] H. S. Kang, C. S. Lee, T. Ree, and F. H. Ree, J. Chem. Phys. 82, pp. 414-422 (1985); J. H. Kim, T. Ree, and F. H. Ree, J. Chem. Phys. 91, pp. 31313147 (1989).

[2] (a) J. D. Weeks, D. Chandler, and H. C. Andersen, J. Chem. Phys. 54, pp. 5237-5247 (1970); (b) H. C. Andersen, J. D. Weeks, and D. Chandler, Phys. Rev. A 4, pp. 1597-1607 (1971).

[3] M. Ross, J. Chem. Phys. 71, pp. 1567-1571 (1979).

[4] H. S. Kang, T. Ree, and F. H. Ree, J. Chem. Phys. 84, pp. 4547-4557 (1986).
[5] H. S. Kang and F. H. Ree, J. Chem. Phys. (1993) to appear.

[6] Y. Choi, T. Ree, and F. H. Ree, J. Chem. Phys. 95, pp. 7548-7561 (1991).

[7] F. H. Ree, J. Chem. Phys. 78, pp. 409-415 (1983).

[8] M. Costantino and S. Rice, J. Phys. Chem. , pp. 9034-9036 (1991).

[9] W. J. Nellis, et al., J. Chem. Phys. 95, pp. 5268-9036 (1991); G. L. Schott, High Pressure Research 6, 187 200 (1991).

[10] F. H. Ree, J. Chem. Phys. 81, pp. 1251- (1984).

[11] F. H. Ree and M. van Thiel, "Effective like- and unlike-pair interactions at high pressure and high temperature," in Shock Compression of Condensed Matter, 1991, New York: North Holland, 1992, pp. 225-228.

[12] N. F. Carnahan and K. E. Starling, J. Chem. Phys. 51, pp. 635-636 (1969).

[13] G. A. Mansoori, N. F. Carnahan, K. E. Starling, and T. W. Leland, Jr., J. Chem. Phys. 54, pp. 15231525 (1971).

[14] P. Ballone, G. Pastore, G. Galli, and D. Gazzillo, Mol. Phys. 59, pp. 275-290 (1986). 

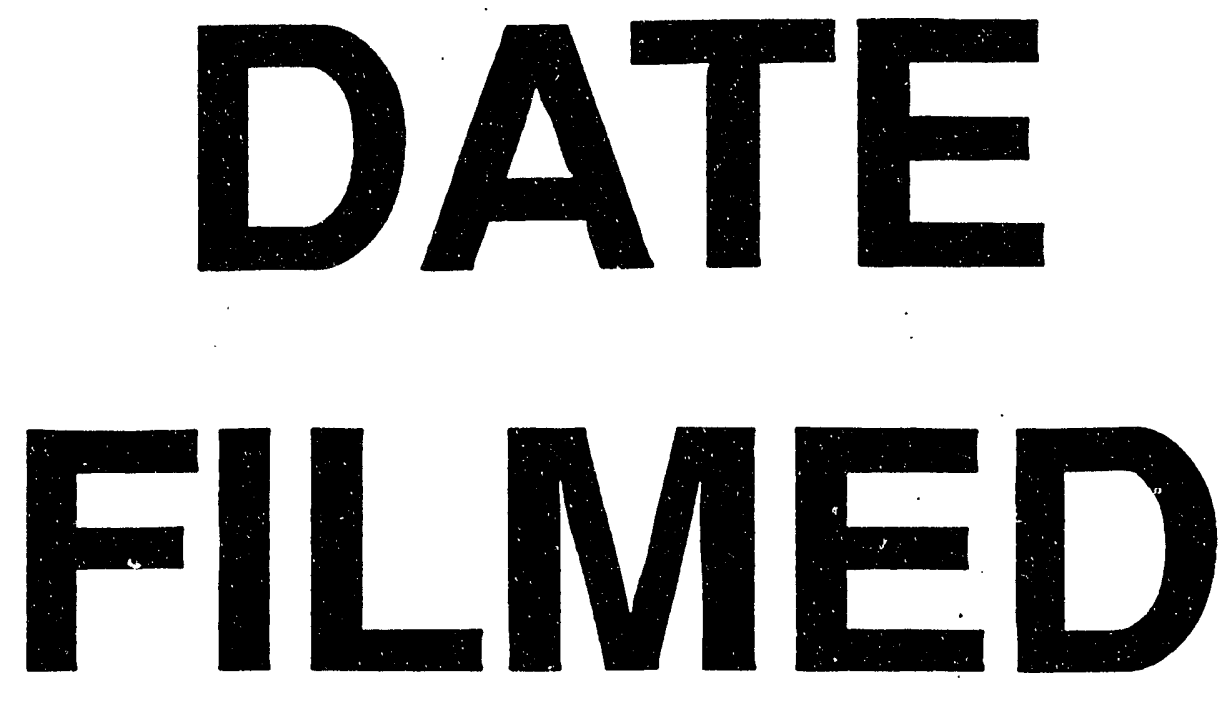

$11 / 9 / 93$
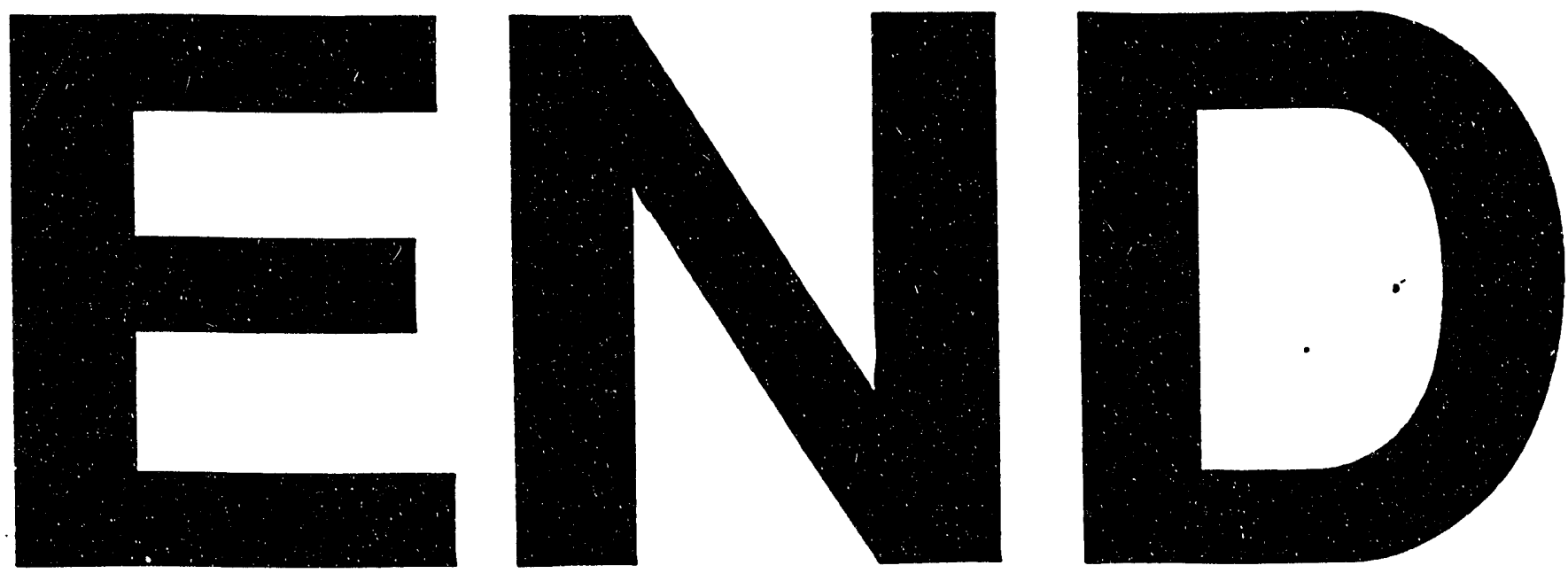
